apuntesuniversitarios.upeu.edu.pe

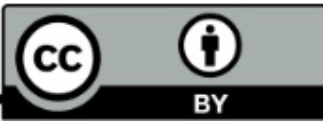

Apuntes Universitarios, 2020: 10(3), julio-setiembre

ISSN: 2304-0335 DOI: https://doi.org/10.17162/au.v10i3.480

\title{
Frecuencia de factores de riesgo para el desarrollo de anorexia y bulimia en un colegio de Lima, 2017
}

\section{Frequency of risk factors that develop anorexia and bulimia in a private educational institution in Lima, Perú, 2017}

\author{
Pilar Gomez-Sanchez ${ }^{1}$, Katherine Gutierrez ${ }^{2}$, Ericson L Gutierrez ${ }^{3 a}$ \\ Universidad de San Martin de Porres. Lima, Perú ${ }^{13}$. \\ Universidad Ricardo Palma. Lima, Perú2. \\ (iD) https://orcid.org/0000-0002-7465-5814 1 \\ (iD https://orcid.org/0000-0003-2285-7614² \\ (iD) https://orcid.org/0000-0003-4725-6284 3
}

Recibido: 23 de julio 2019

Aprobado: 01 de junio de 2020

\begin{abstract}
Resumen
El objetivo de este trabajo de investigación es determinar la frecuencia de factores de riesgo para desarrollar anorexia y bulimia en alumnos de $3^{\circ}$ y $4^{\circ}$ de secundaria de una institución educativa de San Juan de Lurigancho, Lima. Para este propósito, se realizó un estudio transversal. Luego, se aplicó un instrumento validado denominado "factores de riesgo de Anorexia y Bulimia (FRAB)" a los alumnos, durante el mes de octubre del 2017. En el estudio se incluyeron un total de 100 alumnos, del cual el $67 \%$ fueron mujeres. El $21 \%$ de los alumnos, presentaron un mayor riesgo de presentar anorexia y bulimia en comparación con los demás alumnos del plantel. Los principales factores de riesgo fueron conductas influidas por la familia y los pares (25\%), actitudes irracionales (22\%) y autoestima (22\%). En conclusión, la frecuencia de riesgo para presentar anorexia y bulimia en general fue del $21 \%$, los principales factores de riesgo identificados fueron conductas influidas por la familia y los pares, actitudes irracionales y autoestima.
\end{abstract}

Palabras clave: anorexia, bulimia, adolescentes, salud 


\begin{abstract}
The objective of this research work is to determine the frequency of risk factors for developing anorexia and bulimia in students in the 3rd and 4th years of secondary school in an educational institution in San Juan de Lurigancho, Lima. For this purpose, a cross-sectional study was carried out. Then, a validated instrument called "risk factors for Anorexia and Bulimia (FRAB)" was applied to the students, during the month of October 2017. A total of 100 students were included in the study, of which $67 \%$ were women. $21 \%$ of the students had a higher risk of presenting anorexia and bulimia compared to the other students on campus. The main risk factors were behaviors influenced by family and peers (25\%), irrational attitudes (22\%) and self-esteem $(22 \%)$. In conclusion, the risk frequency for presenting anorexia and bulimia in general was $21 \%$, the main risk factors identified were behaviors influenced by the family and peers, irrational attitudes and self-esteem.
\end{abstract}

Keywords: anorexia, bulimia, adolescents, health

\title{
Introducción
}

Los trastornos de la conducta alimentaria (siendo los más representativos la anorexia y bulimia nerviosa) son un alarmante problema de salud pública. Estas afectan a personas cada vez más jóvenes y solo la intervención temprana es la clave para lograr una recuperación completa. Por otro lado, al revisar la literatura reciente sobre la epidemiología, el curso y el resultado de los trastornos de la conducta alimentaria (TCA) de acuerdo con la quinta edición del Manual Diagnóstico y Estadístico de los Trastornos Mentales (DSM-5), se encuentra que la prevalencia a lo largo de la vida de la anorexia nerviosa entre las mujeres podría ser de hasta el 4\%, y de la bulimia nerviosa el 2\% (Smink, Van-Hoeken y Hoek, 2013).

De igual manera, los trastornos alimentarios afectan a países latinoamericanos. En un metaanálisis que incluyó 17 estudios de Argentina, Brasil, Chile, Colombia, México y Venezuela se encontró una tasa media de prevalencia puntual del $0,1 \%$ para la anorexia nerviosa, y del 1,16\% para la bulimia nerviosa; ello evidenció que los trastornos de la alimentación son disturbios mentales cada vez más comunes en América Latina (Kolar, Mejia, Chams y Hoek, 2016).

En el Perú, los TCA son más prevalentes en el área urbana, llegando al $6.0 \%$ en Lima y Callao, donde es más frecuente en mujeres (7.1\%) en comparación de hombres (4.9\%). Realizando una comparación por sexo y tipo de trastorno alimenticio, en las conductas de bulimia se encontró una prevalencia de $1.6 \%$ de mujeres y $0.3 \%$ de hombres. Asimismo, la bulimia nerviosa y la anorexia nerviosa tienen una prevalencia de $0,7 \%$ y $0,2 \%$ en mujeres, no encontrándose casos en varones (Gonzales, 2017). 
Ciertos estudios muestran que la población más vulnerable a estos desordenes son los escolares, tanto hombres como mujeres, esto debido a que son influenciados por los medios de comunicación, comentarios de amigos y/o familiares o llegan a esta enfermedad como consecuencia de una autoestima baja entre otros factores asociados. Por lo tanto, Es importante la prevención y detección precoz de los TCA, ya que las consecuencias tanto físicas, emocionales, como psicológicas son graves e involucra no solo a la persona sino a todo su entorno. En función a lo anteriormente mencionado, se plantea el presente estudio de investigación que tiene como objetivo general determinar los factores asociados al desarrollo de la anorexia y bulimia nerviosa tomando como población a los estudiantes de $3^{\circ}$ y $4^{\circ}$ de secundaria del colegio particular "Corazón de María", del distrito de San Juan de Lurigancho, en Lima, Perú.

\section{Materiales y métodos}

Para los fines de esta investigación se realizó un estudio descriptivo transversal, llevándose a cabo en la Institución Educativa Particular "Corazón de María”, ubicada en el distrito de San Juan de Lurigancho, durante el periodo octubre - diciembre 2017. Se incluyó a la totalidad de alumnos de 3ero y 4to de secundaria, debido a que la población era pequeña y accesible. Se excluyeron a aquellos estudiantes que no desearon participar del estudio y/o no contaron con autorización de sus padres o tutor legal mediante consentimiento informado.

Por otor lado, a fin de determinar los factores de riesgo de anorexia y bulimia se utilizó un instrumento denominado "Factores de riesgo de Anorexia y Bulimia" (FRAB) (Corona, 2013). Este instrumento fue elaborado en México y validado con la participación de 200 jóvenes de sexo femenino entre 11 y 16 años, el mismo rango de edad de la población del presente estudio. Dicho instrumento contiene 8 dimensiones y el valor de alfa de Crombach obtenido en su validación fue de 0,960 .

La primera dimensión "actitudes irracionales" está compuesta por 12 preguntas con un alfa de Crombach de 0,911, seguida de "pensamientos irracionales" con 13 preguntas y un alfa de 0,883; la dimensión "autoestima" presenta 11 preguntas con un alfa de 0,850; la "influencia de medios de comunicación" y "conducta influida de la familia y los pares" están elaborados con 8 y 5 preguntas con un alfa de 0,812 y 0,685 respectivamente; "tendencia a la comparación" y "rasgos de personalidad" conformados por el mismo número de preguntas, 4, con un alfa de 0,654 y 0,710 respectivamente. Finalmente, la dimensión "autocontrol" está conformada por 7 preguntas y presenta un alfa de 0,781 . 
Para la recolección de datos en esta investigación, se solicitó la relación total de estudiantes de 3ero y 4to de secundaria. Posteriormente, el instrumento fue autoadministrado por los alumnos en las aulas de clase, previa autorización del tutor del aula. La autora principal del estudio estaba presente al momento de tomar la encuesta y se asesoraba a los alumnos en caso tenían dudas al momento de llenar la encuesta.

En la población de estudio, el instrumento mostro un valor alfa de Crombach de 0,971 en general y según cada dimensión se halló: "Actitudes irracionales" (0,942), "pensamientos irracionales" (0,831), “autoestima" (0,877), “influencia de medios de comunicación" $(0,829)$, "autocontrol" (0,740), "tendencia a la comparación" (0,715), "conducta influida por la familia y pares" $(0,807)$ y "rasgos de personalidad" $(0,634)$.

Los datos obtenidos fueron ingresados al programa estadístico SPSS V20.0, luego, se elaboraron tablas y gráficos y se determinaron medidas, resumen y tendencia central. Para realizar la comparación de medias de los puntajes con el sexo de los participantes, se aplicó la prueba T Student o la U de Mann-Whitney de ser el caso. Todos los cálculos fueron realizados con un nivel de confianza del 95\%. Para crear los rangos de los puntajes obtenidos para el puntaje general y por cada uno de los factores de riesgo se utilizó la fórmula de Stanones, con lo cual se establecieron 3 categorías, alto, medio y bajo.

El presente estudio fue aprobado por el comité de ética y el departamento de grados y títulos de la Facultad de Medicina de la Universidad San Martin de Porres. Asimismo, se contó con la aprobación escrita del colegio. Todos los participantes fueron informados y aquellos que tuvieron la autorización de sus padres mediante el consentimiento respectivo confirmaron su participación mediante un asentimiento entendido. Los resultados obtenidos solo fueron utilizados para fines del estudio; se aseguró el anonimato y la confidencialidad de los alumnos.

\section{Resultados}

Se incluyó un total de 100 alumnos, de los cuales $67 \%$ pertenecían al sexo femenino y

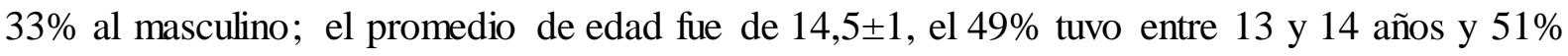
entre 15 y 16 años. $50 \%$ cursaban el tercer grado y $50 \%$ el cuarto grado. El promedio del puntaje total obtenido por los alumnos fue de 97,81 448.07 (rango [29-220]). Al categorizar los puntajes, se obtuvo que el $21 \%$ de alumnos tuvieron puntajes altos en el instrumento de medición (Figura $1)$. 
Figura 1. Puntaje total de riesgo de presentar anorexia y bulimia de acuerdo a su intensidad

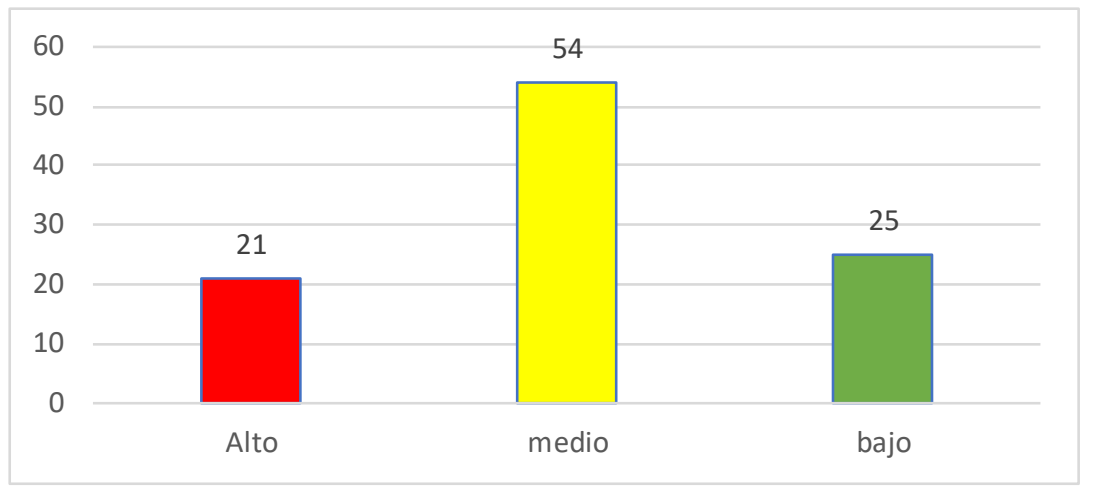

Al categorizar los factores de riesgo para anorexia, se aprecia que las "conductas influidas por la familia y pares," es el factor de riesgo con mayor porcentaje (25\%), seguido de "actitudes irracionales" con (22\%). El factor de riesgo con un menor porcentaje de puntuación fue "rasgos de personalidad" (17\%) (Tabla 1, Figura 2).

Tabla $\mathbf{N}^{\circ} 1$. Promedio de los puntajes obtenidos en cada factor de riesgo

\begin{tabular}{|c|c|c|c|}
\hline Variables & $\mathrm{N}^{\circ}$ de ítems & Media & D.E. \\
\hline Actitudes irracionales & 12 & 18.4 & 12.0 \\
\hline Pensamientos irracionales & 13 & 18.2 & 11.0 \\
\hline Autoestima & 11 & 16.5 & 8.6 \\
\hline Influencia de medios de comunicación & 8 & 12.3 & 6.3 \\
\hline Autocontrol & 7 & 11.5 & 5.1 \\
\hline Tendencia a la comparación & 4 & 6.4 & 3.6 \\
\hline Conductas influidas por la familia y los & & 7.2 & 4.4 \\
\hline pares & 5 & & \\
\hline Rasgos de personalidad & 4 & 7.4 & 3.0 \\
\hline
\end{tabular}

D.E.: Desviación Estándar 


\section{Figura 2. Factores de riesgo para presentar anorexia y bulimia de acuerdo a su intensidad}

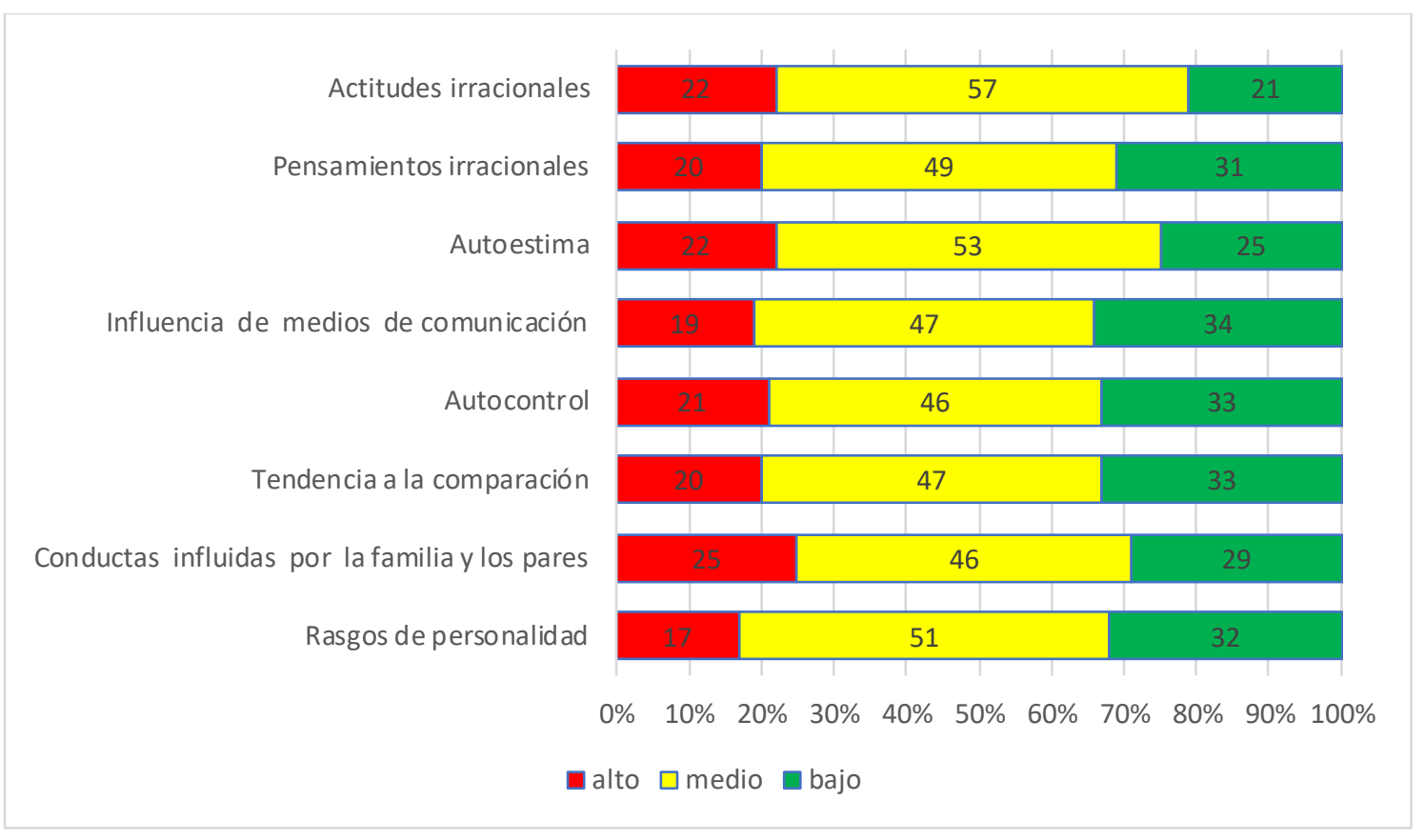

Por último, no se apreció diferencia significativa en el puntaje total (Figura 3), ni en cada uno de los factores de riesgo presentes en el instrumento de medición, de acuerdo al sexo de los adolescentes (Tabla 2).

Figura 3. Media de puntaje total de riesgo de presentar anorexia y bulimia de acuerdo al sexo del estudiante

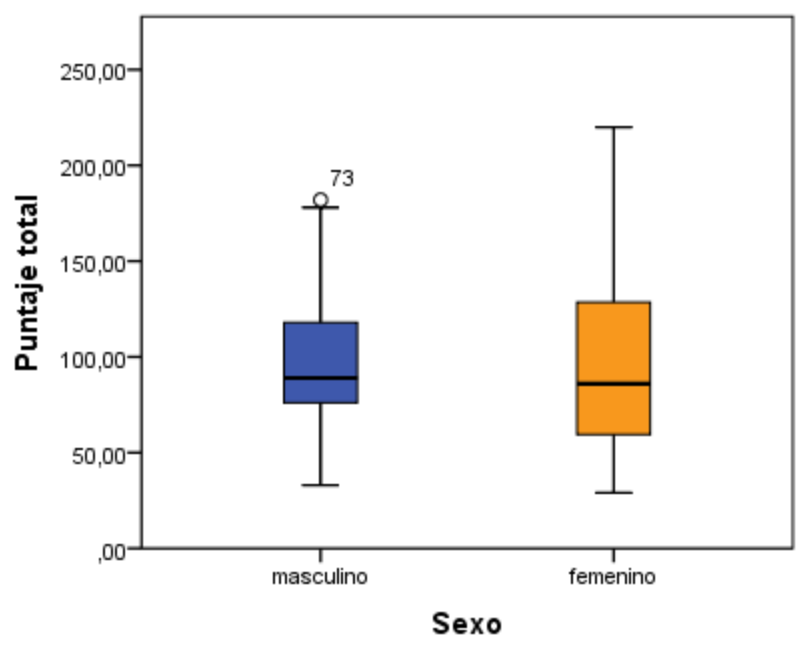




\section{Tabla $\mathrm{N}^{\circ}$ 2. Factores de riesgo de presentar anorexia $\mathrm{y}$ bulimia de acuerdo al sexo del estudiante}

\begin{tabular}{|c|c|c|c|c|c|c|c|}
\hline \multirow{2}{*}{ Factores de riesgo } & \multicolumn{3}{|c|}{ Femenino } & \multicolumn{3}{|c|}{ Masculino } & \multirow{2}{*}{ valor } \\
\hline & $\mathbf{N}$ & Media & D.E. & $\mathbf{N}$ & Media & D.E. & \\
\hline Actitudes irracionales & 67 & 19.18 & 13.05 & 33 & 16.85 & 9.48 & .363 \\
\hline Pensamientos irracionales & 67 & 18.73 & 11.71 & 33 & 17.00 & 9.44 & .462 \\
\hline Autoestima & 67 & 16.28 & 9.13 & 33 & 17.00 & 7.40 & .696 \\
\hline $\begin{array}{l}\text { Influencia de medios de } \\
\text { comunicación }\end{array}$ & 67 & 12.18 & 6.84 & 33 & 12.58 & 5.11 & .769 \\
\hline Autocontrol & 67 & 11.42 & 5.18 & 33 & 11.58 & 5.09 & .886 \\
\hline Tendencia a la comparación & 67 & 6.45 & 3.89 & 33 & 6.27 & 3.08 & .822 \\
\hline $\begin{array}{l}\text { Conductas influidas por la familia y } \\
\text { los pares }\end{array}$ & 67 & 7.21 & 4.59 & 33 & 7.12 & 3.98 & .926 \\
\hline Rasgos de personalidad & 67 & 7.13 & 3.29 & 33 & 7.85 & 2.37 & .269 \\
\hline
\end{tabular}

\section{Discusión}

En el presente estudio se encontró que hasta el $21 \%$ de los alumnos tuvieron un puntaje alto en la encuesta, presentando un mayor riesgo de anorexia y bulimia en comparación de los demás estudiantes del plantel. Asimismo, los principales factores de riesgo encontrados en los alumnos del colegio fueron: la influencia de familia y pares (25\%), seguido de las actitudes irracionales $(22 \%)$. No se encontró diferencia significativa entre los factores de riesgo respecto al sexo.

En cuanto al riesgo de padecer algún trastorno de la conducta alimentaria (TCA), múltiples estudios realizados en Perú, muestran resultados diversos. Un trabajo realizado el año 2003 demostró que el 15.1\% de adolescentes procedentes de colegios de Lima Metropolita na presentaban riesgo de TCA (Martínez Zusman et al, 2003). Posteriormente, en el año 2006, un estudio realizado en estudiantes universitarios de sexo femenino de la Universidad Cesar Vallejo, demostró que el $27 \%$ de las evaluadas tuvo un riesgo psicológico, un $26 \%$ un riesgo social y un $28 \%$ en riesgo sociocultural alto para presentar TCA (Borrego, 2010).

De igual manera, una reciente investigación realizada por Vega et al. (2018) en estudiantes de nutrición de la misma universidad, muestra que el 15,6\% de ellos presentan un mayor riesgo de TCA. (Vega Mosquera y Cubas, 2018). En este contexto, el principal factor de riesgo encontrado en el presente estudio fue la conducta influida por familias y pares $(25 \%)$. 
Está demostrado que la familia influye de manera sustancial en la aparición y el desarrollo de los TCA, por lo cual la Academy for Eating Disorders (AED) de los Estados Unidos, recomienda el tratamiento tanto del paciente como de la familia en sí, para tener un resultado satisfactorio (Le Grange, 2010).

Por otro lado, las actitudes irracionales fueron el segundo factor de riesgo más frecuente presentado en esta población. Un estudio realizado en Sevilla, España, muestra que las creencias irracionales son más frecuentes en mujeres que sufren diagnóstico de anorexia y bulimia, en comparación con controles sanos; asimismo, no hay diferencia entre estas actitudes entre pacientes con AN y Bulimia (Borda, Del Rio y Torres, 2003). Uno de los factores alarmantes es la baja autoestima, llegando a un puntaje de riesgo alto de $22 \%$ en nuestro estudio. Esto también se observó en un estudio realizado por Berenguií, Castejón y Torregrosa (2016) en Murcia, España, donde se encontró que los jóvenes universitarios que presentan un mayor número de factores de riesgo para presentar un TCA, tienen a su vez una menor autoestima.

En el presente estudio el 19\% de los alumnos presentaron riesgos altos para la dimensión de "influencia de los medios de comunicación". Este hallazgo fue reportado por Lazo el al. (2015) en un estudio realizado en tres colegios del distrito de La Victoria (Lima), en el cual se encontró que los medios de comunicación influyen aumentando la probabilidad de presentar un TCA en esta población (Lazo, Quenata y Mayta-Tristan, 2015). La tendencia a la comparación es otro factor influyente en estas patologías presentando un $20 \%$ de los alumnos de esta institución. Un estudio realizado por Alcaraz (2017) en España, muestra que los escolares adolescentes que tenían tendencia a la comparación al momento de realizar ejercicio físico, presentaban mayor frecuencia de síntomas propios de TCA.

Cabe resaltar en el presente estudio se encuentra un $17 \%$ de riesgo alto en lo relacionado a los rasgos de personalidad, el cual entre todos los factores analizados es el de menor porcentaje. Diversos estudios muestran que existe un alto porcentaje de comorbilidad entre trastornos de personalidad y los TCA. Un estudio realizado en México por Molina et al. (2019), muestra que los pacientes con TCA presentan un mayor porcentaje de compulsividad, así como rasgos indicativos de inestabilidad emocional.

Entre las limitaciones del presente estudio se encontró que, por ser un solo colegio, estos resultados no serían necesariamente representativos de la población de Lima. Asimismo, el instrumento utilizado reconoce factores de riesgo sobre anorexia y bulimia, mas no se realiza un diagnóstico. Sin embargo, estos resultados podrían dar una primera aproximación del porcentaje de adolescentes en riesgo de presentar algún trastorno de conducta alimentaria. De igual manera, si el presente instrumento determina factores de riesgo y no necesariamente un 
diagnóstico, los resultados permitirían focalizar a los alumnos con mayor riesgo para aplicar un nuevo instrumento diagnóstico, así como aplicar estrategias de intervención para evitar la aparición de algún trastorno.

En conclusión, en el presente estudio se encontró que el $21 \%$ de los alumnos presentó un mayor riesgo de desarrollar anorexia y bulimia, en comparación con los demás alumnos, siendo los factores de riesgo más importantes "influencia de la familia y los pares," "actitudes irracionales", "autoestima" y "autocontrol". Además, debido a la creciente incidencia que se ha venido observando en la población en general, la anorexia y la bulimia deben ser consideradas un serio problema de salud pública, por lo que se sugiere seguir realizando este tipo de estudios en otras poblaciones para observar de qué manera se manifiestan dichos trastornos, efectuar una detección a tiempo y evitar la gravedad de la enfermedad.

\section{Referencias}

Alcaraz-Ibáñez, M. (2017). Comparación social de la apariencia en contextos de ejercicio físico como variable predictora de los trastornos de la conducta alimentaria en adolescentes de ambos sexos. Espiral. Cuadernos del Profesorado, 10(21), 80-89.

Berenguíi, R., Castejón, M., y Torregrosa, M. (2016). Insatisfacción corporal, conductas de riesgo para trastornos de la conducta alimentaria en universitarios. Revista mexicana de trastornos alimentarios, 7(1), 1-8.

Borda, M., Del Rio C., y Torres I. (2003). Creencias irracionales en mujeres con anorexia nerviosa y bulimia nerviosa. Med Psicosom, 65, 19-23.

Borrego, C. (2010). Factores de riesgo e indicadores clínicos asociados a los trastornos de la conducta alimentaria. Rev Psicol, 12, 13-50.

Corona M (2013). Validez y confiabilidad de un instrumento para medir factores de riesgo para anorexia y bulimia (Tesis para Licenciatura). Instituto Tecnológico de Sonora, Obregón Sonora, México.

Gonzales, F. (2017). Situación de salud en los adolescentes y jovenes en el Perú. Lima, Perú, URL disponible en http//bvs.minsa.gob.pe/local/MINSA/4143.pdf

Kolar, D., Mejía, D., Chams, M., y Hoek, H. (2016) Epidemiology of eating disorders in Latin America: a systematic review and meta-analysis. Current Opinion in Psychiatry, 29(6), 363-71.

Lazo,Y., Quenaya, A., y Mayta-Tristan, P. (2015). Mass media influence and risk of developing eating disorders in female students from Lima, Peru. Archivos Argentinos de Pediatría, 113(6), 519-525. 
Le Grange, D., Lock, J., Loeb, K., y Nicholls, D. (2010). Academy for Eating Disorders position paper: the role of the family in eating disorders. Int J Eat Disord, 43(1), 1-5.

Martinez, P., Zusman, L., Hartley, J., Morote, L., y Calderon, A. (2003). Estudio epidemiológico de los trastornos alimentarios y factores asociados en Lima Metropolitana. Revista de Psicología de la PUCP, 21(2), 234-69

Molina-Ruiz, R., Alberdi-Páramo, Í., Castro Oller, M., Gutiérrez, N., Carrasco, J., y DíazMarsá, M. (2019). Personalidad en pacientes con trastorno alimentario en función de la presencia/ausencia de comorbilidad con trastorno límite de la personalidad. Revista mexicana de trastornos alimentarios, 10(1), 109-120.

Smink, FR., Van Hoeken, D., y Hoek, HW.(2013). Epidemiology, course, and outcome of eating disorders, Curr Opin Psychiatry, 26(6), 543-8

Vega, E., Mosquera, Z., y Cubas, F. (2018). Frecuencia de trastornos alimentarios en estudiantes de la escuela de nutrición de la Universidad César Vallejo Lima este, 2017. UCV-Scientia Biomedica, 1(2), 80-84. 